Historic, Archive Document

Do not assume content reflects current scientific knowledge, policies, or practices. 



\subsection{4}

B. IHALSEY SPENCER
GLADIOLUS GROWER

97 Hopkins Place,

Longmeadou, Hass.

WHOLESALE SPRING 1929

TERMS: Prepaid delivery on ali orders cver $\$ 3.00$. On smaller orders add 10\%. Cash with order or $25 \%$ holds for spring delivery.

5 bulbs at ten rate; 25 at hundred rate. Special prices on bulblets in quantity. No substitution without permission. Pint at Quart rate.

ALL STOCK IS GUARANTEED TRUE AND HEALTHY. IIBERAL COUNT AND EXTRAS. AIL STJCK HAS BTEN GROMN FRON YOUNG BULBS AND BULBLETS THAT HAVT BEEN TREATED TACH YEAR AND I WILI PLACE THE RUN OF OUUAITY ALONG SIDE ANY BULBS PRUDUCED. EXPARIENCED BULB MEN MHO HAVE SHEN THIS STOCK IN STCRAGE DURING THE FALE AND WINTER FAVE FRANILY STATED THAT IT IS THE CLEANYST AND FINEST RUN THEY HAVE SEEN. I KNOW IT IS GOOD, AS NOTHING ELSE WILI GO HERE AND NONE OF II BOUGHT IN.

I am going to greatly reace number of varieties on my list. Ask for special list with closing out prices.

\section{SATISFACTION GUARANTEED}

VARIFTIRS RECOMMENDED: Anie Lamrie; Catherine CoJeman; Ethlyn; Elkhart; Fern Kyle; Elorense(special on smail stock) Gaiety; Golden Drear; Helga; J V B; Los Angeles; Lustre; Orange Flame; JEAN DU TAILLES; PRIDE CF MANAKAH. Saraband.

Have fine stoct of these in small sizes especially and. bulblets. Also Remembrance for cut flower work. 

BULBS per 100 unless otherwise noted

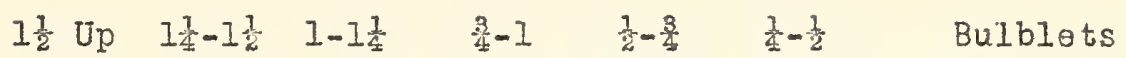

No. 1 No. 2 No. 3 No. 4 No. 5 No. $6 \quad 100 \quad 1000$ Q⿱亠䒑十.

Ah Pink and yellow

$\$ 4.00 \quad \$ 3.00 \quad \$ 2.00 \quad \$ 1.50 \quad \$ 1.00 \quad \$ .50$

A E Kundred per I Pink

$.50 \quad .25 \$ .15$

Albania White

.50

$\begin{array}{llll}3.00 \quad 2.00 \quad & 1.50 \quad 1.00\end{array}$

.15

2.50

Alton Orange

4.00

4.00

3.00

2.00

1.50

.80

$3.00 \quad 2.00$

Alice Tiplady Saffron

4.00

3.00

$\begin{array}{lll}2.00 & 1.50 & 1.00\end{array}$

Anna Eberius Purplo

$18.00 \quad 16.00$

Anthony Zonker

Arabia or Persia "Black

Balboa each

\section{Glad"}

B I Smith Lavender

Butterboy Yellow

Capt Boynton Lavender

$14.00 \quad 11.00 \quad 9.00$

6.00

3.00

2.00

1.50

1.00

$6.00 \quad 5.00$

4.00

3.00

1.50

.70

.50

.35

2.50

1.50

.75

2.50

2.00

1.50

1.00

.75

6.00

5.00

4.00

3.00

.75

1.25

1.00

2.00

.50

Carmen Sylva White

2.50

1.00

$17.00 \quad I 4.00 \quad I I .00$

8.00

3.00

2.00

.75

.20

.50

.50

2.50

.35

.50

Claremont Yellow

Crimson Glow Crimson

Cynthia Clough per 10

4.00

3.20

2.50

2.00

1.50

5.00

1.00

.80

Apricot

2.00

1.75

I. 50

1.25

1.00

.80

.50

Diana Red

$4.00 \quad 3.20$

3.00

2.00

1.00

Dorothy McKibben Rose Pink 3.00

2.00

Dr Flkins Tall white

Dr F E Bennett Orange Scarlet

Dr N Shook each Rose red .40

.35

2.50

1.50

7.00

4.00

.75

3.00

.20

.10

2.00

I B Tilliamson Purple

E G Hill True Pink

3.00

2.00

1.00

.70

.40

$1.00 \quad .50$

II J Shaylor Rose Pink

4.00

3.00

2.00

1.50

1.50

2.00

1.50

1.25

1.00

2.40

1.80

1.20

.75

IIf White

3.20

2.4 .0

1.60

.60

.80

Ethelyn Orange Yellow

6.00

4.00

2.50

1.50

3.00

2.50

1.50

1.00

.75

Exquisite Am. Beauty

3.20

2.40

1.60

1.00

4.00

3.00

2.00

1.50

.30

1000

Elkhart Purple

Forn Kyle Cream

6.00

5.00

4.00

3.00

2.00

1.00

Florence per 10 Lavender

Fontaine Light pink

2.50

2.00

1.50

1.00

.75

.50

Frosty Red flaked gray

4.00

3.20

2.40

2.00

1.25

.75

6.00

4.50

3.00

2.00

2.50

2.00

1.50

1.00

.50

$\$ 3.00$

$\begin{array}{rrr} & & 2.00 \\ & .50 & 3.00 \\ & & 1.50 \\ & & 1.50 \\ & 5.00 & 20.00 \\ & .75 & 6.00 \\ & & \\ & .75 & 3.00 \\ & 1.00 & \\ & .50 & 1.50 \\ & .50 & 1.50 \\ & .80 & 6.00 \\ .35 & 1.50 & 4.00 \\ & .50 & 2.00 \\ & 5.00 & 20.00 \\ .30 & 2.00 & \\ & .50 & 1.00\end{array}$

1.00

1.00

$.75 \quad 3.00$

3.00

$.50 \quad 1.00$

$.50 \quad 2.00$

$.50 \quad 1.00$

.80

$1.00 \quad 3.50$

$2.00 \quad 7.00$

$.75 \quad 4.00$

$.75 \quad 4.00$

$2.00 \quad 5.00$

$.80 \quad 3.50$

$2.00 \quad 5.00$

Gaiety Rose Pink \& White

3.00 
BULBS per 100 unless otherwise noted

\begin{tabular}{llllllll}
$I \frac{1}{2}$ Up & $1 \frac{1}{4}-1 \frac{1}{2}$ & $1-1 \frac{1}{4}$ & $\frac{3}{4}-1$ & $\begin{array}{c}\frac{1}{2}-\frac{3}{4} \\
\frac{1}{4}-\frac{1}{2}\end{array}$ & Bulblets \\
No. 1 & No. 2 & No. 3 & No. 4 & No. 3 & No. 6 & $100 \quad 1000$ & Qt. \\
\hline
\end{tabular}

\begin{tabular}{|c|c|c|c|c|c|c|c|c|c|}
\hline Fold Yellow & $\$ 5.00$ & $\frac{5}{8}=.00$ & $\$ 3.00$ & $\$ 2.00$ & $\$ 1.00$ & $\$ 75$ & & $\$ .75$ & $\$ 3.00$ \\
\hline GIadness Lavender & 3.00 & 2.50 & 2.00 & 1.50 & 1.00 & .50 & & .50 & 1.00 \\
\hline Giant Nymph Light Pink & 6.00 & 5.00 & 4.00 & 3.00 & 2.00 & 1.00 & & .85 & 3.25 \\
\hline GoIden Heasure Yellow & 5.00 & 4.00 & 3.00 & 2.00 & 1.50 & .80 & & & 5.00 \\
\hline Golden Gleam & 3.00 & 2.00 & & & & & & & 1.00 \\
\hline Solden Dream Deep Yellow. & \pm 0.00 & 30.00 & 25.00 & 18.00 & 14.00 & 12.00 & 2.50 & 20.00 & spec. \\
\hline Golden Frills per 10 & 3.00 & 2.50 & 2.00 & 1.50 & 1.00 & .50 & 1.50 & 12.00 & \\
\hline Golden Salmon per 10 & 3.50 & 3.00 & 2.50 & & & & 2.00 & & \\
\hline olden Eagle Early yellow & 40.00 & 30.00 & 25.00 & 18.00 & 14.00 & 10.00 & 2.50 & 20.00 & Spec. \\
\hline Ioriana Golden salmon & 30.00 & 25.00 & 20.00 & 35.00 & 10.00 & 5.00 & 1.00 & 9.00 & \\
\hline elga Early Salmon & 3.00 & 2.50 & $? .00$ & 1.50 & 1.00 & .50 & & .50 & 2.00 \\
\hline indian Surmer Lavender & 6.00 & 5.00 & 4.00 & 3.00 & 2.00 & 1.00 & & 1.00 & 5.00 \\
\hline Illuminator Red & 6.00 & 5.00 & & & 2.00 & 1.00 & & 2.50 & \\
\hline Imrnensity Salmon & 6.00 & $5.00^{\circ}$ & & & 2.00 & 1.00 & & 1.50 & \\
\hline Ina Ta.II pink & 20.00 & 16.00 & 12.00 & 10.00 & 7.00 & 4.00 & & 3.00 & \\
\hline Jewell Rose \& yellow & 2.50 & 2.00 & 1.50 & 1.00 & .75 & .50 & & & 1.00 \\
\hline Jacoba Von Beyren Purple & 6.00 & 5.00 & 4.00 & 3.00 & 2.00 & 1.00 & & .75 & 3.00 \\
\hline J du Tailles per 10 Pink & 2.00 & 1.75 & 1.50 & 1.00 & .75 & .50 & & 3.00 & 10.00 \\
\hline Joergs White, per 10 & 6.00 & 5.50 & 4.00 & 3.50 & & & & & \\
\hline ilac Wonder & 7.00 & 6.00 & 5.00 & 4.00 & 3.00 & 2.00 & & 3.00 & \\
\hline ongfellow per 10 Pink & 2.50 & 2.00 & 1.75 & 1.50 & 1.00 & .75 & 1.00 & 5.00 & \\
\hline Ios Angeles Shrimp pink & 6.00 & 5.00 & 3.00 & 2.015 & 1.00 & .75 & & 1.00 & 5.00 \\
\hline Louise Lavender & 4.00 & 3.20 & 2.50 & 2.00 & 1.50 & .80 & & & \\
\hline Lustre Glorious red & 6.00 & 5.50 & 5.00 & 3.75 & 2.50 & 1.50 & & & 3.00 \\
\hline Uary Frey each Lavender & 0.00 & 5.00 & 4.00 & 3.00 & 2.00 & & 25.00 & & \\
\hline Ladison Cooper Garnet & 5.00 & 4.00 & 3.00 & 1.50 & 1.00 & .50 & & & 2.00 \\
\hline If Kunderd Early white & 7.00 & 6.00 & $\leq .50$ & 3.00 & 2.00 & 1.00 & & & 3.50 \\
\hline llarietta Salmon & 8.00 & 7.00 & 6.00 & 5.00 & 3.00 & 2.00 & & 2.00 & \\
\hline Larnia Golden Orange & 10.00 & 8.00 & 6.00 & 4.00 & 3.00 & 1.50 & & 3.00 & 14.00 \\
\hline Hildred Adeline Salmon & 6.00 & 5.00 & 1.00 & 3.00 & 2.00 & 1.00 & & 2.00 & \\
\hline Ining Toy Bupf & 3.00 & 2.50 & 2.00 & 1.50 & 1.00 & .50 & & & 1.00 \\
\hline Minuet each Lavender & 1.50 & 1.35 & 1.20 & 1.00 & .80 & .60 & & & \\
\hline Iniss lhadison Light pink & 6.00 & 5.00 & 4.00 & 3.00 & 2.00 & 1.00 & & 1.50 & 5.00 \\
\hline Helba per 10 Cream pink & 1.50 & 1.25 & 1.00 & .75 & .50 & .30 & 1.00 & & \\
\hline Honon Bright pinis & 7.00 & 6.00 & 5.00 & 4.00 & 3.00 & 2.00 & & & 7.50 \\
\hline Mrs Norton Pink \& Cream & 3.60 & 3.20 & 2.50 & $\approx .00$ & 1.50 & .80 & & .50 & 2.00 \\
\hline Lirs Horaberger per 10 Whte & e 6.00 & 5.00 & 4.00 & 3.00 & 2.00 & 1.00 & & 12.00 & \\
\hline Hes Douglas Begonia Rose & 7.00 & 6.00 & 5.00 & 4.00 & 2.00 & 1.00 & & 1.00 & 5.00 \\
\hline Lírs Sisson por 10 Cam pink & $=3.50$ & 3.00 & 2.00 & 1.50 & .75 & .50 & .50 & & \\
\hline Mrs Killion per 10 White & 2.25 & 2.00 & J. .75 & 1.50 & 1.00 & .60 & 1.00 & & \\
\hline Muriel Lavender & 6.00 & 5.00 & 4.00 & 3.00 & 1.50 & .75 & & 1.00 & \\
\hline Opalescent Light Lav Pink & $=6.00$ & & & & 1.0 & .50 & & .50 & 2.50 \\
\hline Odin Salmon & 4.00 & 3.50 & 3.00 & 2.50 & 1.00 & .50 & & .50 & 2.00 \\
\hline Orange Flam orange & 10.00 & 8.00 & 6.00 & 4.50 & 2.50 & 1.50 & & 2.00 & 5.00 \\
\hline
\end{tabular}



BULBS per 100 unless othermise noted

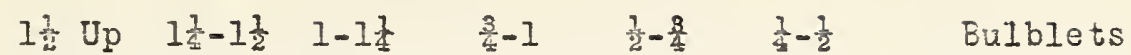

No. 1 No. 2 No. 3 No. 4 No. 5 No. $6 \quad 1001000$ Qt.

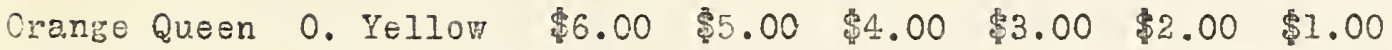

$\begin{array}{llllll}\text { Orchid Lady each Lavender } 1.00 & .90 & .75 & .60 & .50 & .40\end{array}$

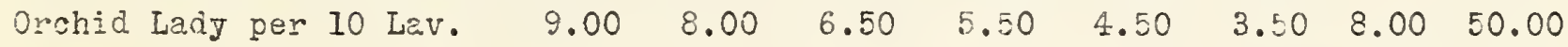

Furamount Pinks

Pavl Piitzer, each Purple

Park Wanakah Lose 12.00

Princely whito

Durple Butterfly

Purple Glory

Pythia Glorious Red

Quinton eariy Coral pink

Red Fire

Remembrance Salmon Rose

Richard Diener Pink

Rita Beck per 10 Pink 10.00

Rose Ash

rosemery per 10 Lavender

Rose lisist old Rose

Rosy Glow Pink

Salmon Buf f

Saraband Bordeaux

Scerlet Princeps

Scerlot Wonder

Shell Pink

Sidney Plummer Yellow

Sweet Lavender

$\mathrm{Str}$ Rose per 10 Rose Pink

Svea Pink \& yellov Prim

10.00

6.00

7.00

6.00

.45

10.00

8.00

5.00

6.00

5.00

6.00

.40

8.00

6.00

4.00

5.00

4.00

5.00

4.00

3.00

2.00

5.00

4.00

3.00

2.00

1.50

1.50

1.00

2.00

4.00

3.00

9.00

8.00

6.50

3.00

2.00

1.50

1.25

1.00

$15.00 \quad 13.00$

11.00

5.00

4.00

.75

9.00

3.00

1.00

5.00

1.00

.50

7.00

2.00

$\begin{array}{rr}3.00 & 2.00 \\ 25.00 & 15.00\end{array}$

3.00

2.00

13.00

1.50

11.00

4.00

6.00

5.00

3.00

1.00

7.00

.75

1.50

4.00

2.00

2.50

2.00

3.00

2.00

1.50

.75

2.00

1.50

2.50

1.50

1.00

2.00

4.00

3.00

6.00

5.00

4.00

3.00

2.00

4.00

3.00

4.00

3.00

$\therefore .00$

2.00

1.50

1.00

1.00

.85

.75

.60

5.00

.50

.80

1.00

.40

.80

.50

$3.00 \quad 2.00$

1.50

1.00

.50

.50

Uh]an Chier Parly Red

5.00

4.00

5.00

4.00

12.00

9.00

6.00

3.00

2.00

1.50

3.00

.75

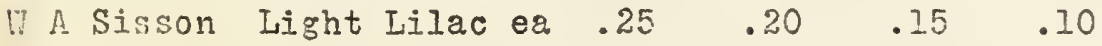

$\$ .50 \$ 3.50$

5.00

3.00

5.0015 .00

3.5012 .00

2.0010 .00

1.00

$.50 \quad 3.00$

$1.50 \quad 5.00$

$.50 \quad 2.00$

$.50 \quad 1.50$

$.50 \quad 5.00$

7.0060 .00

$.50 \quad 1.50$

1.00

$1.00 \quad 6.00$

$1.00 \quad 3.00$

$.50 \quad 1.00$

$1.00 \quad 10.00 \quad 40.00$

$.50 \quad 1.00$

$.50 \quad 4.00$

.80

$.50 \quad 2.50$

$.50 \quad 2.50$

3.00

1.00

$1.25 \quad 5.00$

$.50 \quad 1.00$

$.50 \quad 1.50$

$.50 \quad 1.50$

2.50

.50

Yellow Monder per 10

2.50

2.00

1.50

1.00

.75

.50

$1.00 \quad 8.00$

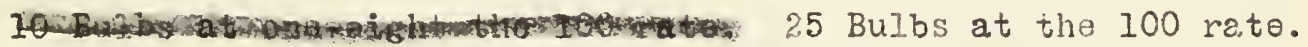


